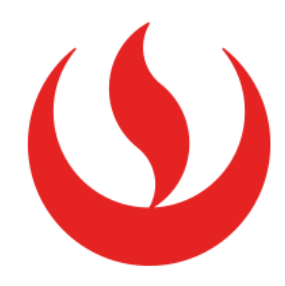

UNIVERSIDAD PERUANA DE CIENCIAS APLICADAS

\title{
FACULTAD DE CIENCIAS DE LA SALUD
}

PROGRAMA DE NUTRICIÓN Y DIETÉTICA

Número de controles prenatales y su asociación con el bajo peso al nacer en mujeres de 15 a 49 años según la Encuesta Demográfica y de Salud Familiar ENDES-2016

\section{TESIS}

Para optar el título profesional de: Licenciado en Nutrición y Dietética

\section{AUTORES}

Gonzales Huamán, Flor de María Susan (0000-0003-0258-3871)

Saavedra Grandez, Wilmar Alexis (0000-0003-2398-8771)

\section{ASESORA DE TESIS}

Liria Domínguez, María Reyna (0000-0002-5637-1519)

Lima, 12 de Noviembre de 2018 
A nuestros padres, Guillermina y Gumercindo, Martha y Abiud, por su amor, ejemplo de superación e inspiración para nosotros y apoyo incondicional a lo largo de nuestras vidas y carrera universitaria. A los amigos verdaderos que nos acompañaron en las buenas y en las malas 


\section{Agradecimientos}

Le damos gracias a nuestros padres, Guillermina y Gumercindo, Martha y Abiud, por apoyarnos en todo momento, por los valores que nos han inculcado, y por habernos dado la oportunidad de tener una excelente educación en el transcurso de nuestra vida. Sobre todo, por ser ejemplos de vida a seguir. Agradecemos a nuestra asesora de tesis por sus conocimientos vertidos en este trabajo y en nosotros mismos, sin ello no hubiera sido posible la realización de esta tesis 


\section{RESUMEN}

Introducción: El BPN está asociado a diversos factores (socioeconómicos, culturales, condiciones biológicas de la madre) y diversas patologías que afectan tanto a la madre como al feto. Este bajo peso tiene consecuencias inmediatas y pueden prevalecer hasta la vida adulta. Una de las estrategias descritas para prevenir el BPN o identificar a tiempo problemas con el feto/niño y la madre es el control prenatal. Objetivo: Evaluar la asociación de BPN y número de controles prenatales en madres de 15 a 49 años basados en la ENDES-2016. Metodología: Estudio transversal de análisis secundario de la base de datos de la ENDES2016. Se evaluó el peso al nacer de 16062 niños. La variable de respuesta fue BPN en la última gestación, que independientemente de la edad gestacional, se consideró a todo niño con un peso al nacer menor de $2500 \mathrm{~g}$. La variable de exposición de interés es el número de controles prenatales durante la última gestación (0-5 controles, 6-7 controles, 8-10 controles, $>11$ controles). Esta variable ha sido categorizada según las recomendaciones del Ministerio de Salud (MINSA) (mínimo 6 controles), Organización Mundial de la Salud (OMS) (mínimo 8 controles) y American College of Obstetricians and Gynecologists (ACOG) (mínimo 11 controles). Las variables de control se incluyeron: edad, estado civil, nivel educativo, quintil de riqueza, departamento, área de residencia, orden de nacimiento, talla materna, abortos, periodo intergenésico, suplementación con hierro y metros sobre el nivel del mar (m.s.n.m). Para evaluar diferencias en el análisis bivariado se usó chi cuadrada para variables categóricas y T - Test para variables numéricas. A través de regresión de Poisson se calculó la razón de prevalencias cruda $(\mathrm{RPc})$ y ajustada $(\mathrm{RPa})$. Resultados: A medida que aumenta el rango de controles prenatales disminuye el riesgo de BPN tanto en el modelo crudo (6-7 controles: RPc=0,65, IC 95\%: 0,53-0,80; 8-9 controles: RPc=0,26, IC 95\%: 0,21-0,31; 11 a más controles: $\mathrm{RPc}=0,22$, IC 95\%: 0,18-0,28), como en el modelo ajustado (6-7 controles: $\mathrm{RPa}=0,65$, IC 95\%: 0,53-0,81; 8-9 controles: $\mathrm{RPa}=0,25$, IC 95\%: 0,21-0,32; 11 a más controles: $\mathrm{RPa}=0,23$, IC 95\%: 0,18-0,29). Conclusión: El presente estudio encontró que el aumento en el número de controles prenatales disminuye el BPN. Se sugiere incorporar la recomendación de número de controles prenatales dada por la OMS.

Palabras clave: Bajo peso al nacer, BPN, control prenatal, cuidado prenatal, neonatos. 


\section{ABSTRACT}

Introduction: LBW is associated with several factors (socioeconomic, cultural, biological conditions of the mother) and various pathologies that affect both the mother and the fetus. This low weight has immediate consequences and can prevail until adult life. One of the strategies to prevent LBW or identify early problems with the fetus/child and the mother is prenatal control. Objective: To evaluate the association of LBW and number of prenatal controls in mothers from 15 to 49 years based on the ENDES-2016. Methodology: Crosssectional study of secondary analysis of the ENDES-2016 database. A total of 21175 births were found, of which 16062 children were selected for the study. The response variable was BPN in the last gestation, that regardless of the gestational age, every child with a birth weight of less than $2500 \mathrm{~g}$ was considered. The variable of exposure of interest is the number of prenatal controls during the last gestation (0-5 controls, 6-7 controls, 8-10 controls,> 11 controls). This variable has been categorized according to the recommendations of the Ministry of Health (MINSA) (minimum 6 controls), World Health Organization (WHO) (minimum 8 controls) and American College of Obstetricians and Gynecologists (ACOG) (minimum 11 controls). In the case of control variables were included: age, marital status, educational level, wealth quintile, department, area of residence, birth order, maternal height, abortions, intergenesic period, iron supplementation and meters above the level of sea. To evaluate differences in the bivariate analysis, chi square was used for categorical variables and T- Test for continuous quantitative variables. Through Poisson regression the crude prevalence ratio (RPc) and adjusted prevalence ( $\mathrm{RPa}$ ) were calculated. Results: The prevalence of LBW decreases according to the increase in prenatal controls: from 0 to 5 (18.42\%), from 6 to 7 (11.97\%), from 8 to $10(4.70 \%)$ and from 11 to more $(4.14 \%)$ (p $<0.001)$. In addition, as the range of prenatal controls increases, the risk of low birth weight decreases in both the crude model (6-7 controls: $\mathrm{RPc}=0.65,95 \% \mathrm{CI}: 0.53-0.80,8-9$ controls: $\mathrm{RPc}=0.26,95 \%$ CI: 0.21-0.31, 11 more controls: $\mathrm{RPc}=0.22,95 \%$ CI: 0.18-0.28) as adjusted (6-7) controls: $\mathrm{RPa}=0.65,95 \% \mathrm{CI}$ : 0.53-0.81, 8-9 controls: $\mathrm{RPa}=0.25,95 \%$ CI: 0.21-0.32, 11 more controls: $\mathrm{RPa}=0.23,95 \% \mathrm{CI}$ : 0.18-0.29). Conclusion: The present study found that the increase in the number of prenatal controls decreases LBW. $\}$

Key words: Low birth weight, LBW, prenatal control, prenatal care, neonates. 


\section{TABLA DE CONTENIDO}

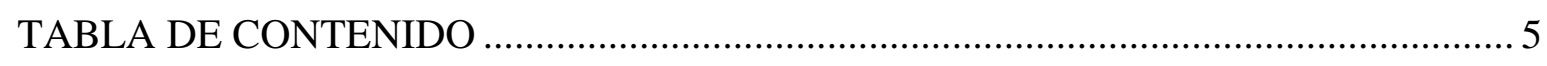

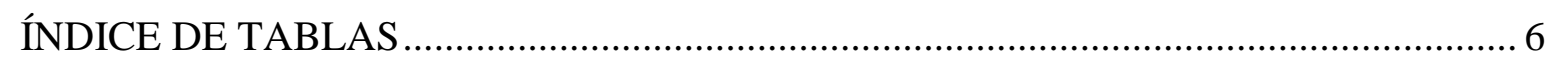

ÍNDICE DE IMÁGENES......................................................................................... 7

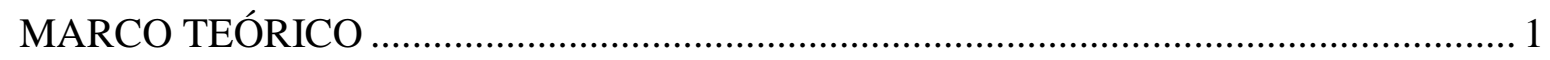

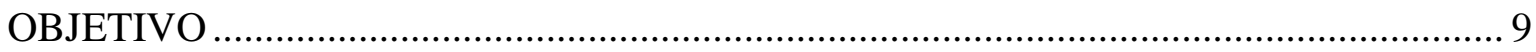

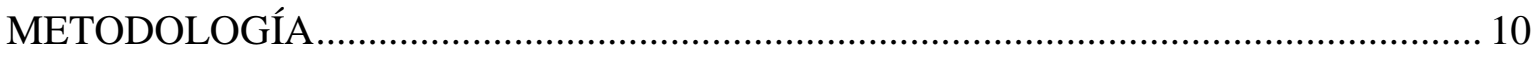

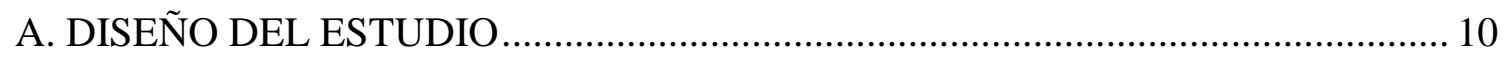

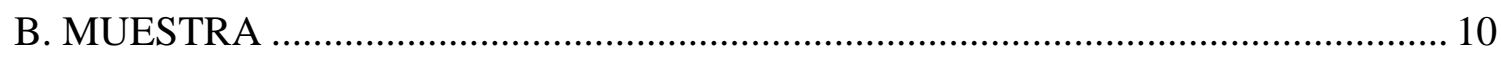

C. CRITERIOS DE INCLUSIÓN Y EXCLUSIÓN ........................................................ 10

Criterios de inclusión............................................................................................... 10

Criterios de exclusión ............................................................................................. 10

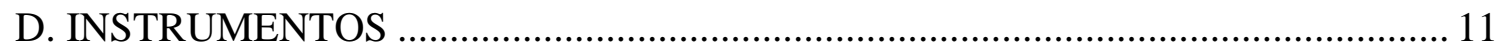

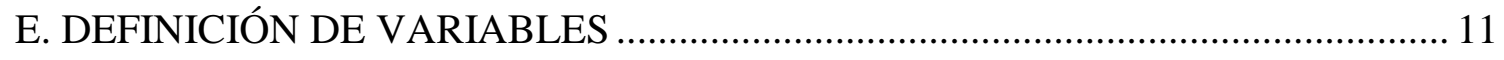

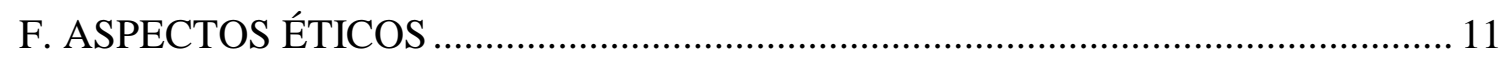

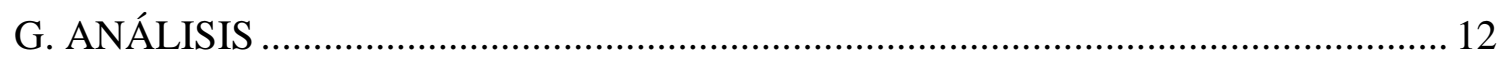

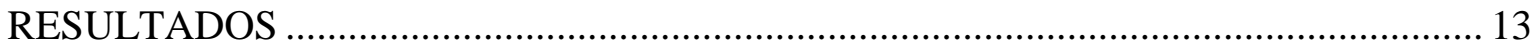

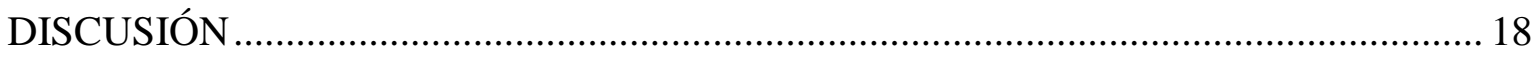

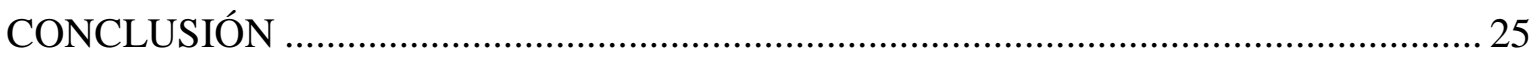

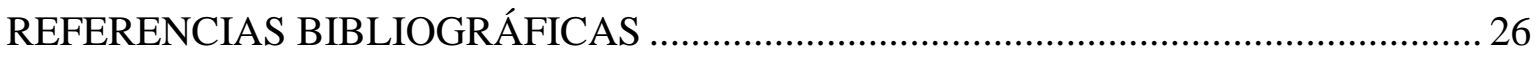

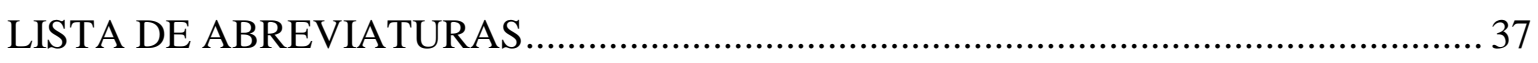

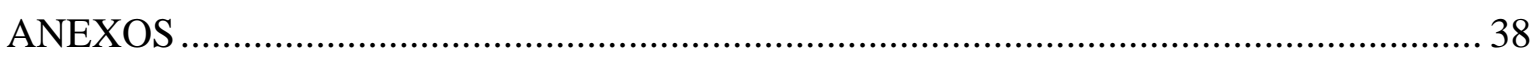




\section{ÍNDICE DE TABLAS}

Tabla 1. Características sociodemográficas y secundarias, ENDES-2016 ...................... 15

Tabla 2. RP crudo y ajustado de asociación entre número de controles prenatales y bajo

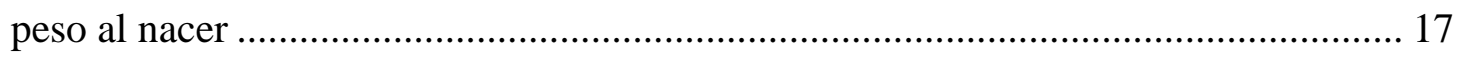




\section{ÍNDICE DE IMÁGENES}

Figura 1. Flujograma de la población estudiada...................................................... 13 


\section{MARCO TEÓRICO}

Según la Organización Mundial de la Salud (OMS), se define como bajo peso al nacer (BPN) al neonato que pese menos de $2500 \mathrm{~g}(\mathbf{1}, \mathbf{2})$. A nivel mundial, en el año 2011, se reportó un 15\% de BPN (3), mientras que a nivel de América, en el año 2016, la Organización Panamericana de la Salud (OPS), reportó un 8,0\% de BPN y para Perú 7,0\% (4).

Diversos estudios han evidenciado que el BPN tiene consecuencias inmediatas que podrían prevalecer hasta la vida adulta. Estos problemas aumentan a medida que el peso al nacer del niño es menor $(\mathbf{5}, \mathbf{6}, \mathbf{7}, \mathbf{8})$. Las consecuencias pueden ser diversas y afectar de diferente manera de acuerdo a la edad. Así tenemos que, el BPN aumenta el riesgo de morir durante los primeros meses y años, debido a diferentes causas; tales como las infecciones respiratorias agudas (IRAs), enfermedades diarreicas agudas (EDAs), alteraciones del sistema inmunológico, entre otros. Si bien estas enfermedades son más prevalentes en la primera infancia, en niños que nacieron con BPN el riesgo se puede duplicar. Se ha descrito que las IRAs en estos niños se deben a factores anatómicos (mayor calibre de las vías aéreas mayores y vías aéreas más periféricas son de mucho menor calibre, lo cual favorece el ingreso de bacterias y otros agentes y la oclusión de estas, respectivamente) sumado a que podrían aún estar inmaduros (en prematuros) algunos de los mecanismos de defensa contra las infecciones $(\mathbf{6 , 9}, \mathbf{1 0}, \mathbf{1 1})$. Lo mismo sucede con las EDAs, los BPN son más susceptibles y éstas suelen ser más severas que en niños con un peso adecuado al nacer $(\mathbf{1 0}, \mathbf{1 1})$. Los niños con BPN, además, pueden tener un sistema inmunológico deprimido, debido a la menor concentración de inmunoglobulinas y de deficiente actividad de macrófagos y linfocitos $(\mathbf{1 1}, \mathbf{1 2})$. De acuerdo al estudio de Álvarez et al, entre el 13 y el $24 \%$ de los BPN padecen trastornos neurológicos así como entre el 6 y el $13 \%$ déficit intelectual; lo cual repercute en la adaptación social y calidad de vida desde la niñez hasta la adultez (13). En el caso de los pequeños para la edad gestacional (longitud y/o peso al nacimiento <-2 DE), sigla PEG, la recuperación de la talla es infrecuente y las secuelas neurológicas suelen ser mayores (14). El desarrollo normal del cerebro requiere de una adecuada oxigenación, nutrición y equilibrio hormonal, además de evitar sustancias tóxicas. El sistema endocrino, el más importante para el crecimiento fetal, involucra los factores de crecimiento similares a la insulina (IGF-I). Aunque el papel de la IGF-I cerebral en la fisiología neural no está 
claro, algunos investigadores formulan la hipótesis de que la deficiencia prolongada de IGFI inducida por la desnutrición fetal en el útero puede afectar el desarrollo normal del sistema nervioso central o estar relacionado con una hipoglucemia grave, lo que resulta en un funcionamiento intelectual más bajo de los individuos $(\mathbf{1 5}, \mathbf{1 6}, \mathbf{1 7}, \mathbf{6})$. Los PEG experimentan un crecimiento recuperador espontáneo postnatal (catch-up growth). Sin embargo, cuando éste es muy rápido puede predisponer a posterior resistencia a la insulina, acúmulo de adiposidad central, pubertad adelantada y rápida, mayor riesgo de desarrollar diabetes tipo 2 y patología cardiovascular en la edad adulta $(\mathbf{6}, \mathbf{7}, \mathbf{8}, \mathbf{9}, \mathbf{1 7})$.

El BPN está asociado a diversos factores de riesgo: socioeconómicos, culturales, condiciones biológicas de la madre y diversas patologías que afectan tanto a la madre como al feto. De acuerdo con el Comité para estudiar la prevención del BPN (Committee to Study the Prevention of Low Birth Weight), los riesgos se clasificaron de la siguiente manera: (i) Riesgos demográficos: edad (menor de 20 años, más de 34 años), ser soltero, bajo nivel educativo, bajo nivel socioeconómico, etnicidad; (ii) Riesgos médicos anteriores al embarazo: orden de nacimiento (primer hijo o más de 4), baja talla e IMC (Índice de Masa Corporal) materna, abortos, anomalías genitourinarias y del tubo neural, diabetes, hipertensión, partos con BPN, factores genéticos maternos relacionados con peso al nacer; (iii) Riesgos médicos en el embarazo actual: intervalo corto entre embarazos, embarazo múltiple, pobre ganancia de peso, hipotensión, hipertensión, preeclampsia-eclampsia, infecciones (bacteriuria, rubéola y citomegalovirus), sangrado en el primer o segundo trimestre, problemas de placenta (placenta previa, ruptura de placenta), hiperémesis, oligohidramnios/polihidramnios, anemia, isoinmunización, anomalías fetales, cuello uterino incompetente; (iv) Riesgos conductuales y ambientales: vivir en zonas muy altas sobre el nivel del mar, fumar, estado nutricional inadecuado, abuso de alcohol y otras sustancias, exposición a agentes tóxicos; (v) Riesgos de atención médica: cuidado prenatal ausente o inadecuado, prematuridad $(\mathbf{1 8}, \mathbf{1 9})$.

La edad de la madre es considerada un factor de riesgo, principalmente, en aquellas mujeres muy jóvenes o añosas primerizas (20). Mientras más joven es la gestante tiene mayor probabilidad de tener hijos prematuros o con BPN, estos resultados pueden deberse a la inmadurez biológica, la cual tiene dos aspectos: por un lado se encuentra la inmadurez ginecológica joven $(6,7)$ y por otra, el concebir un nuevo ser antes de haber completado su 
propio crecimiento. A medida que las madres tienen hijos más tarde, aumenta la posibilidad de BPN, esto se explica por la coexistencia de padecimientos anteriores a la gestación y gestacionales asociados a trastornos a nivel del miometro. Esta situación predispone a sufrir una mayor tasa de complicaciones perinatales, entre las cuales se encuentra, además del BPN, el RCIU (Retardo en el Crecimiento Intrauterino: bajo peso para la edad gestacional), aumento de mortalidad materna, perinatal e infantil (6).

Se ha reportado muchas veces la asociación entre el nivel socioeconómico y el peso al nacer $(\mathbf{2 1}, \mathbf{2 2})$. Tal es así que en el trabajo de Rahman et al, encontraron que era menos probable que los niños nacieran con BPN en familias de mayor nivel socioeconómico (tercil más alto) en comparación con los más pobres (tercil más bajo) (23). Asimismo, un mayor nivel educativo influye en los conocimientos de la madre sobre los cuidados prenatales y sobre una adecuada alimentación. Además, un mejor nivel educativo de los progenitores podría permitir gozar de una mayor estabilidad económica, mayor solidez matrimonial y una mejor atención prenatal (6). También, se ha encontrado asociación del incremento de BPN y el estado civil "soltera" $(\mathbf{2 4}, \mathbf{2 5})$.

La paridad también influye en el BPN, esto puede deberse a que los nacimientos previos se asocian con cambios en la anatomía materna, que podrían influir con la eficiencia en el peso al nacer, una mujer que tiene experiencia previa de embarazo y nacimiento es capaz de tener a hijos sanos con más eficiencia (6). Por otro lado, las madres que han tenido tres partos o más elevan el riesgo de tener nacimientos con bajo peso (26). Además, el periodo intergenésico entre embarazos es muy importante, ya que en éste y la etapa de lactancia disminuyen los recursos biológicos y nutritivos, por lo que la madre necesita un tiempo de recuperación y preparación para una nueva gestación. Si el tiempo de distancia entre un embarazo y otro es corto (menor de 24 meses), el riesgo de BPN aumenta $(\mathbf{6 , 2 6 ) .}$

Además, las mujeres que presentan enfermedad hipertensiva en el embarazo, también tienen mayor riesgo de BPN, esto debido a las alteraciones en la vascularización de la placenta lo que se traduce en una disminución del riego útero-placentario, que provoca envejecimiento precoz de la placenta, teniendo como consecuencia eclampsia y desprendimiento de la placenta. Ambos pueden provocar la terminación del embarazo antes de llegar a término del mismo. Además, esta afección influye en la nutrición del niño dentro de la placenta, provocando RCIU o un futuro nacimiento con BPN (6). Así mismo, se ha descrito que 
alrededor del 5\% de los hijos de madres diabéticas presentan RCIU; sin embargo aún se desconocen las causas $(\mathbf{2 7}, \mathbf{2 8})$.

La historia obstétrica de los embarazos previos de una mujer es de gran importancia; tal es así que en el estudio Bakketeig et al, menciona que si en el primer parto se tuvo BPN o prematuro conlleva que un segundo parto tenga similares resultados (29). Así mismo, se ha descrito que las mujeres que han tenido dos abortos a más tienen mayor probabilidad de BPN (30).

Otro riesgo médico como el embarazo gemelar tiene como producto más del $10 \%$ de los nacidos pretérminos, los cuales suelen tener un BPN. La existencia de dos fetos a la misma vez en el claustro materno provoca la competencia por la inserción placentaria en busca de mayor oportunidad de nutrición, por lo que se requiere el doble de condiciones en relación con el embarazo único (31).

La anemia es otro factor que se asocia a BPN y prematuridad. La anemia ferropénica es la anemia más frecuente y se incrementa con el embarazo. Esto se debe principalmente a la mayor utilización de hierro en esta etapa (26). Las gestantes necesitan este mineral para la reposición de las pérdidas basales a causa del aumento del tamaño de los eritrocitos y la nutrición del feto, placenta y tejidos (32). La deficiencia de hierro en el embarazo causa alteraciones en el transporte de oxígeno, lo que repercute en la fisiología fetal y por lo tanto en su peso (26).

Los riesgos conductuales y ambientales como fumar pueden afectar el crecimiento fetal. Tal es así que en el estudio de Hamulka et al, se encontró que tanto el tabaquismo como el consumo de alcohol aumentaron el riesgo de parto prematuro junto con el BPN (33).

En el caso de la altitud, en el estudio de Zahran et al, mencionan que el bajo peso se podría dar por la deprivación materna de oxígeno, la cual puede inducir hipoxia hipobárica limitando el crecimiento del feto. Así mismo, se podría dar la posibilidad de un segundo mecanismo que involucra la vía de la glucosa, donde las concentraciones de glucosa arterial y venosa umbilical eran más bajas a gran altitud (2500 m.s.n.m), que resultaba en un menor suministro y consumo de glucosa por parte del feto $(\mathbf{3 4}, \mathbf{3 5})$. Resultados similares también se encontraron en el estudio de Villamonte et al, donde menciona que la hipoxia crónica a causa de la altura produce disminución del peso al nacer; sin embargo, también cita que en 
otros estudios se encontró que en las ciudades con mayor altura se presenta mayor pobreza, siendo esto un efecto negativo en el crecimiento fetal (36).

A nivel nacional, según la ENDES (Encuesta Demográfica y de Salud Familiar) del año 2016, el BPN se asocia a ciertas características de la población, así tenemos que fue mayor en: madres adolescentes menores de 20 años $(8,3 \%)$, población con mayor nivel de pobreza $(9,1 \%)$, sin educación $(9,8 \%)$, mayor número de hijos (con seis y más hijos: 9,9\%), en los que viven en área rural $(8,1 \%)$, en la sierra $(8,1 \%)$ y selva $(8,0 \%)$ y los departamentos de Loreto (10,9\%), Pasco $(9,6 \%)$, Ucayali $(9,4 \%)$ y Cajamarca $(9,1 \%)(37)$.

Una de las estrategias del Ministerio de Salud (MINSA) para la reducción del BPN es la suplementación profiláctica de ácido fólico, hierro y calcio con el fin de evitar problemas en el desarrollo del tubo neural; detectar la anemia y brindar tratamiento oportuno; y prevenir complicaciones de hipertensión, complicaciones en el embarazo y la eclampsia (38). Existen normas que avalan estas disposiciones, tal es así la Norma Técnica de Salud (NTS) para el manejo terapéutico y preventivo de la anemia en niños, adolescentes, mujeres gestantes y puérperas, la cual tiene como objetivo contribuir al desarrollo y bienestar de niños, adolescentes, mujeres gestantes y puérperas en el marco de la atención integral de salud (39, 40). En esta NTS se abarca como medidas de prevención de anemia en la gestación la educación nutricional y la suplementación de la gestante y la puérpera; en el caso del parto se considera el pinzamiento y corte tardío del cordón umbilical e inicio de la lactancia materna. Además de otras medidas como la promoción de consumo de alimentos fortificados con hierro y el consumo de agua segura, el lavado de mano y la higiene de los alimentos en el hogar (40).

Otra estrategia del MINSA para disminuir la mortalidad materna, sus complicaciones, el BPN, entre otros; es la Estrategia Sanitaria de Salud Sexual y Reproductiva en su articulado de Salud Materna y Neonatal. Según la NTS para la Atención Integral en Salud Materna (NTS N ${ }^{\circ}$ 105-MINSA/DGSP.V.01), la atención integral de salud materna se abarca desde la atención preconcepcional, seguido de la atención prenatal reenfocada hasta el plan de parto institucional y el puerperio. Es en esta NTS, donde se indica que la atención prenatal reenfocada es un proceso multidisciplinario de vigilancia y evaluación de la madre embarazada y su hijo, la madre debería recibir un total de 6 atenciones prenatales para considerarse una gestante controlada y es ideal que la primera atención se lleve a cabo antes 
de las catorce semanas de gestación. Esto con el fin de detectar oportunamente factores de riesgo y signos de alarma. Además, enfocar la educación para el autocuidado y la participación de la familia, así como para el manejo adecuado de las complicaciones (39). Esta atención prenatal reenfocada debe ser llevada a cabo en todos los establecimientos de salud por personal calificado (médico gineco - obstetra, médico cirujano, obstetra, dependiendo del nivel de complejidad) (41). En el primer nivel de atención el primer o segundo control prenatal debe ser realizado por un médico ginecólogo-obstetra o médico cirujano; mientras que en el segundo o tercer nivel debe hacerlo un médico gineco-obstetra (39). En la atención prenatal se realiza la evaluación integral del estado actual nutricional (examen físico, evaluación del estado nutricional inicial, ganancia de peso durante el embarazo, medición de la altura uterina); estado emocional (signos de estrés, signos de maltrato y violencia, apoyo familiar) y evaluación odontoestomatológica (42). Es en la primera consulta donde el personal de salud realiza la medición antropométrica (peso y talla) y en las siguientes se realiza en control de ganancia de peso (39). Sin embargo, en una gran parte de los establecimientos de salud de primer nivel no se cuenta con un médico, sólo con enfermera, obstetra y/o técnico de enfermería.

Por su parte, la OMS en su nuevo modelo de atención prenatal indica que la mujer gestante debe tener ocho contactos con profesionales de la salud (43). Ya que una mayor regularidad de contactos de la madre con el sistema de salud se asocia a una disminución de probabilidad de mortalidad en la etapa prenatal. Estos ocho controles permiten mayor oportunidad de detectar posibles problemas, además de mayor comunicación entre personal de salud y la gestante (44). En este modelo de atención prenatal se sugiere que el primer contacto se lleve a cabo hasta las 12 semanas de gestación y sugiere que los posteriores sean a las 20, 26, 30, 34, 36, 38 y 40 semanas (45). La OMS recomienda que diferentes profesionales, como trabajadores de salud no especializados, auxiliares de enfermería, enfermeros, parteras y médicos participen en la prestación de atención prenatal (43).

Por otro lado, la American College of Obstetricians and Gynecologists (ACOG) recomienda la atención anteparto mediante un programa integral de atención. Este programa contiene un enfoque coordinado de la atención médica, la evaluación contínua de los riesgos y el apoyo psicosocial de la madre, idealmente comenzando antes de la concepción con una visita previa; extendiéndose hasta el periodo posparto $(\mathbf{4 6}, \mathbf{4 7})$. En el caso de la atención prenatal 
la ACOG indica que se da a partir del primer trimestre del embarazo, con visitas cada 4 semanas durante las primeras 28 semanas de embarazo, cada 2 a 3 semanas entre 28 y 36 semanas, y semanalmente hasta el parto (entre 11 a 14 visitas de acuerdo al número de semanas de gestación final). Los pacientes con embarazos de alto riesgo o aquellos con complicaciones continuas generalmente se ven con más frecuencia, dependiendo de las circunstancias clínicas $(\mathbf{4 6}, \mathbf{4 7}, \mathbf{4 8}, \mathbf{4 9})$. En el primer control a menos que no haya una visita previa a la concepción, se recopila una base de datos obstétrica, evaluación de riesgos y la educación del paciente; en los siguientes controles o visitas de rutina se evalúa la presión arterial, el peso, el tamaño uterino de la mujer y la consistencia con la fecha estimada del parto, y la presencia de actividad cardíaca fetal. Así mismo, en la evaluación de rutina se realiza el análisis de orina para albúmina y glucosa. También se incluyen psicoprofilaxis, en donde se incluyen temas como: la elección del pediatra/neonatólogo o médico que brinde la atención del recién nacido, información sobre parto anticipado y/o prematuro, las opciones de atención intraparto, los bancos del cordón umbilical, lactancia materna, la elección de un método anticonceptivo posparto y la preparación para el alta hospitalaria $(\mathbf{4 6}, \mathbf{4 7})$.

La ACOG ha establecido durante mucho tiempo el estándar de atención para la práctica obstétrica en los Estados Unidos. Sin embargo, algunos estados de este país como Indiana, Arizona, entre otros han estratificado o usado diferentes nomenclaturas de los niveles de atención materna. La atención materna se refiere a todos los aspectos de anteparto, intraparto y atención postparto de la mujer embarazada. El sistema de clasificación propuesto para la atención del embarazo, parto y puerperio es la siguiente; centro de nacimiento (atención periparto a mujeres de bajo riesgo con embarazos a largo plazo sin complicaciones con una presentación en vértice que se espera que tengan un parto sin complicaciones), atención básica (nivel I - atención a mujeres con bajo riesgo y se espera que tengan un parto sin complicaciones), atención especializada (nivel II - atención a mujeres embarazadas de alto riesgo, pueden ser tanto admitidas como transferidas a la instalación), atención de subespecialidad (nivel III - brindan todos los servicios de nivel I y nivel II, y cuentan con subespecialistas disponibles en el sitio, para ayudar a brindar atención para enfermedades maternas y fetales más complejas) y centros de atención de salud perinatal regionales (nivel IV - incluyen las capacidades de las instalaciones de nivel I, nivel II y nivel III con capacidades adicionales y una experiencia considerable en el cuidado de las mujeres 
embarazadas más complejas y en estado crítico a lo largo de la atención preparto, intraparto y posparto) $(\mathbf{5 0 , 5 1 )}$.

Además, diversos estudios encontraron que el número de controles prenatales se asocia con el resultado del nacimiento. En el estudio de Henderson, se encontró que las mujeres que no pudieron recibir atención prenatal tenían casi tres veces más probabilidades de tener un bebé con BPN que las mujeres que sí lo hicieron (52). La investigación de Tayebi et al, también encontró que las probabilidades de BPN son más altas en mujeres con atención prenatal inadecuada ( $<4$ controles prenatales), por lo que el riesgo es tres veces mayor que el de los otros grupos, adecuado (5-6 controles) e intensivo ( $>6$ controles) (53).

No completar todos los controles prenatales dificulta las actividades de prevención y de promoción de la salud, ya que se limita la interacción entre el profesional de la salud y la futura madre (54). Según ENDES-2016, de los últimos hijos nacidos en los cinco años anteriores a la encuesta, el $88,9 \%$ recibieron de seis a más atenciones prenatales y este fue mayor en área urbana (90,5\%) que en área rural (84,3\%) (37). Sin embargo, pese al porcentaje elevado de la población con control prenatal, hay 7,0\% de recién nacidos con BPN y existe poca información sobre la asociación entre número de controles prenatales y el BPN. 


\section{OBJETIVO}

Evaluar la asociación de BPN y número de controles prenatales en madres de 15 a 49 años basados en la ENDES-2016. 


\section{METODOLOGÍA}

\section{A. DISEÑO DEL ESTUDIO}

Se realizó un estudio transversal secundario. Se utilizó la base de datos de la ENDES-2016 realizada en las 24 regiones del Perú y la provincia constitucional del Callao llevada a cabo por el Instituto Nacional de Estadística e Informática (INEI). El diseño de la ENDES fue bietápico, estratificado e independiente; cuyo objetivo principal fue "proveer información actualizada sobre la dinámica demográfica y el estado de salud de las madres y niños menores de cinco años que permita estimar los indicadores presupuestales, así como la evaluación y formulación de los programas de población y salud familiar en el país" (55).

\section{B. MUESTRA}

Se realizó un cálculo de tamaño de muestra considerando el estudio de Pinzón-Rondón A et al realizado en Colombia, donde se encontró que el promedio de número de visitas prenatales en el grupo de nacidos con BPN fue menor que en el grupo de nacidos con adecuado peso al nacer; 6,12 (DS=3,08) y 6,79 (DS=2,77), respectivamente (56). Así mismo, considerando un nivel de confianza de $99 \%$, razón entre tamaños muestrales de 1 y una potencia estadística de $99 \%$ se obtuvo un tamaño muestral de 3360 personas, calculados con el programa Epidat. Cabe mencionar que el total de mujeres en la ENDES-2016 fue de 34131.

\section{CRITERIOS DE INCLUSIÓN Y EXCLUSIÓN}

\section{Criterios de inclusión}

Se incluyeron a todas las mujeres en edad fértil (MEF) que tuvieron al menos un hijo y que además tengan información del peso al nacer del último hijo nacido vivo y número de controles prenatales, además de todas las variables de interés.

\section{Criterios de exclusión}

Madres con partos gemelares o múltiples.

Nacimientos con 4000 g o más 


\section{INSTRUMENTOS}

La ENDES, a través de una encuesta, ejecutada por el personal de campo previamente capacitado para la realización del estudio, recolecta la información demográfica y de salud. Se cuenta para su realización con Supervisor, Entrevistador y Antropometrista. Durante la encuesta se pesaron y midieron a las MEF usando equipos estandarizados (57).

\section{E. DEFINICIÓN DE VARIABLES}

La variable de respuesta fue BPN en la última gestación, que independientemente de la edad gestacional, se consideró a todo niño con un peso al nacer menor de 2500 g. La variable de exposición de interés es el número de controles prenatales durante la última gestación (0-5 controles, 6-7 controles, 8-10 controles , >11 controles). Esta variable ha sido categorizada teniendo en cuenta las recomendaciones de MINSA (mínimo 6 controles), OMS (mínimo 8 controles) y ACOG (mínimo 11 controles). En el caso de las variables de control se incluyeron: (i) riesgos demográficos; edad (15-19, 20-34 y 35-49 años), estado civil (soltera/divorciada/viuda/separada y casada/conviviente), nivel educativo (sin educación/primaria, secundaria y superior), quintil de riqueza, departamento (Amazonas, Áncash, Apurímac, Arequipa, Ayacucho, Cajamarca, Provincia Constitucional del Callao, Cusco, Huancavelica, Huánuco, Ica, Junín, La Libertad, Lambayeque, Lima, Loreto, Madre de Dios, Moquegua, Pasco, Piura, Puno, San Martín, Tacna, Tumbes y Ucayali), área de residencia (rural y urbana); (ii) riesgos médicos anteriores al embarazo: orden de nacimiento (primer nacimiento, segundo nacimiento, tercer nacimiento, cuarto nacimiento y quinto a más nacimientos), talla materna (menor a $145 \mathrm{~cm}$ y mayor a $145 \mathrm{~cm}$ ), abortos (presencia y ausencia); (iii) riesgos médicos actuales en el último embarazo: periodo intergenésico (menor a 24 meses y mayor o igual a 24 meses), (iv) riesgos conductuales o ambientales: suplementación con hierro (presencia y ausencia), m.s.n.m (<1000, 1000-1999, 2000-2999, 3000-3999, $\geq 4000$ ).

\section{F. ASPECTOS ÉTICOS}

Nuestro estudio se basa en análisis de una base secundaria con acceso público (ENDES2016), en la cual no se divulga información que permita conocer la identidad de los participantes ni la ubicación de sus hogares. Este estudio fue exonerado por el Comité de 
Ética de la Universidad Peruana de Ciencias Aplicadas (UPC) con carta ${ }^{\circ}$ CEI 025 PI 020-18.

\section{G. ANÁLISIS}

El análisis de los datos se realizó utilizando el software STATA 15.0 (StataCorp LLC,Texas, US). Para el análisis descriptivo, las variables categóricas se presentan en porcentajes y las variables cuantitativas en media y desviación estándar. Para evaluar diferencias en el análisis bivariado se usó chi cuadrada para variables categóricas y T Test para variables cuantitativas continuas. A través de regresión de Poisson se calculó la razón de prevalencias cruda (RPc) y ajustada (RPa). Se corroboró ausencia de colinealidad usando del factor de inflación de la varianza (vif<3 en Stata). Los datos fueron analizados tomando en cuenta el diseño de estudio (ponderado por estrato y cluster, usando el comando svy de Stata). Para evaluar diferencias se usó un nivel de confianza del $95 \%$. 


\section{RESULTADOS}

Se encontró un total de 21175 nacimientos en la base de datos de la ENDES-2016, de los cuales se seleccionaron para el estudio 16062 niños. Se excluyeron a aquellos que no contaban con información completa: 1030 niños no tenían información sobre peso al nacer; 2950 niños no contaban con información de control prenatal, 151 niños fueron producto de embarazo múltiple y 982 niños no tenían información completa de las otras variables.

Figura 1. Flujograma de la población estudiada

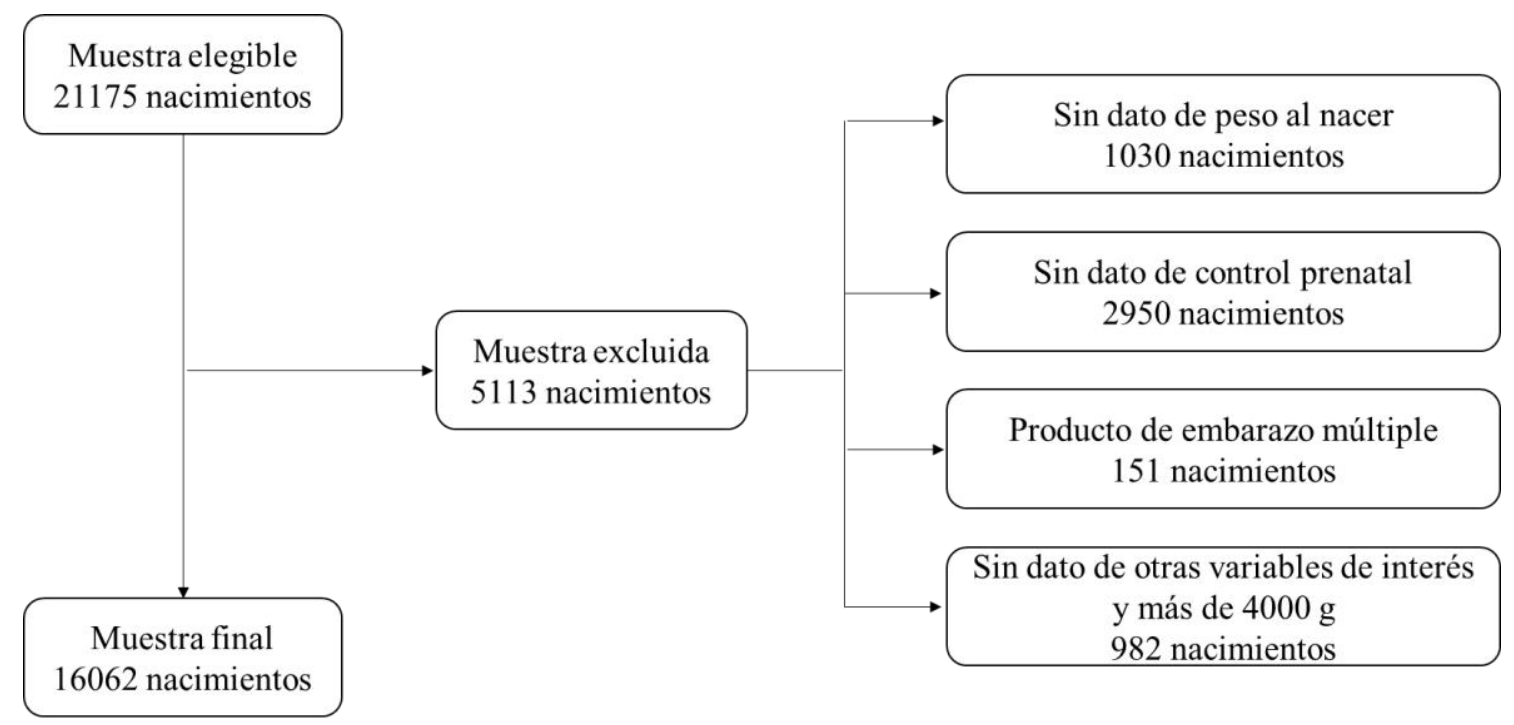

El resultado en promedio de peso al nacer fue: $3179 \mathrm{~g}$ (IC 95\%: 3169-3190), el 92,74\% presentó normopeso y el 7,60\% bajo peso.

La prevalencia de BPN disminuye de acuerdo al aumento de controles prenatales: De 0 a $5(18,42 \%)$, de 6 a $7(11,97 \%)$, de 8 a $10(4,70 \%)$ y de 11 a más $(4,14 \%)(\mathrm{p}<0,001)$.

De los riesgos demográficos, se tiene que las mujeres con menos de 20 años y aquellas mayores de 35 años tienen mayor porcentaje con 7,95\% y 7,92\% respectivamente $(\mathrm{p}=0,0615)$. En cuanto al estado conyugal, las mujeres que no tienen pareja presentan mayor prevalencia con $8,33 \%$ ( $\mathrm{p}=0,1323$ ), en relación al nivel educativo, aquellas que no recibieron educación o que alcanzaron el nivel primario presentan mayor porcentaje con $8,82 \%$ ( $\mathrm{p}=0,0239)$. Las mujeres que pertenecen a un quintil de riqueza muy pobre 
presentan mayor prevalencia con 9,21\%, seguidas de las que se encuentran en el cuarto quintil 7,84\% ( $\mathrm{p}=0,0048)$. Se observa que existe mayor porcentaje en los departamentos: Loreto (11,46\%), Ucayali $(9,39 \%)$, Pasco $(9,15 \%)$, Cajamarca $(8,93 \%)$, Tumbes $(8,82 \%)$ y La Libertad $(8,63 \%)(\mathrm{p}=0,0014)$. El mayor porcentaje con respecto al área de residencia se presenta en el área rural con $8,16 \%(\mathrm{p}=0,0421)$.

De los riesgo médicos anteriores al embarazo, se observa que el orden de nacimiento que mayor prevalencia presenta es del quinto a más con $10,44 \%$, seguido del cuarto $8,28 \%$ y el tercero $7,42 \%(\mathrm{p}=0,0015)$. Las mujeres con talla menor a $145 \mathrm{~cm}$ presentan mayor porcentaje con $11,04 \%$ ( $\mathrm{p}<0,001)$ y las que abortaron al menos una vez presentan mayor prevalencia con $8,83 \%(\mathrm{p}=0,0859)$.

De los riesgos médicos actuales en el último embarazo, el periodo intergenésico menor a 24 meses presenta mayor prevalencia con 9,45\% ( $\mathrm{p}=0,0291)$.

De los riesgos conductuales y ambientales, aquellas que no se suplementaron con hierro tienen mayor porcentaje con $10,33 \%(\mathrm{p}=0,0007)$ y con respecto a la altitud la prevalencia va en aumento a mayor metros sobre el nivel del mar: menos de 1000 (6,89\%), de 1000 y $1999(7,31 \%)$, de 2000 a $2999(7,38 \%)$, de 3000 a $3999(8,52 \%)$ y mayor o igual a 4000 $(10,14 \%)(\mathrm{p}=0,0587)$. 
Tabla 1. Características sociodemográficas y secundarias, ENDES-2016

\begin{tabular}{|c|c|c|c|}
\hline \multirow[b]{2}{*}{ Variables } & \multicolumn{2}{|c|}{ Peso al nacer } & \multirow[b]{2}{*}{ Valor $P$} \\
\hline & $\begin{array}{c}\text { Normo peso } \\
n=14,896\end{array}$ & $\begin{array}{l}\text { Bajo peso } \\
\mathrm{n}=1,166\end{array}$ & \\
\hline Total de nacimientos & 92,74 & 7,60 & \\
\hline Controles prenatales & & & $<0,001$ \\
\hline De 0 a 5 & 81,58 & 18,42 & \\
\hline De 6 a 7 & 88,03 & 11,97 & \\
\hline De 8 a 10 & 95,30 & 4,70 & \\
\hline De 11 a más & 95,86 & 4,14 & \\
\hline \multicolumn{4}{|l|}{ Riesgos demográficos } \\
\hline Edad de la madre al nacimiento (media \pm IC $95 \%$ ) & $\begin{array}{c}30,07(29,91- \\
30,22)\end{array}$ & $\begin{array}{c}30,15(29,62- \\
30,67)\end{array}$ & 0,0615 \\
\hline Menos de 20 años & 92,05 & 7,95 & \\
\hline De 20 a 34 años & 93,29 & 6,71 & \\
\hline De 35 a 49 años & 92,08 & 7,92 & \\
\hline Estado conyugal & & & 0,1323 \\
\hline Soltera/ Divorciada/separada/viuda & 91,67 & 8,33 & \\
\hline Casada / Conviviente & 92,94 & 7,06 & \\
\hline Nivel de educación & & & 0,0239 \\
\hline Sin educación / Primaria & 91,18 & 8,82 & \\
\hline Secundaria & 93,08 & 6,92 & \\
\hline Superior & 93,24 & 6,76 & \\
\hline Quintil de riqueza & & & 0,0048 \\
\hline $\begin{array}{l}\text { Quintil inferior (Extremadamente } \\
\text { pobre) }\end{array}$ & 90,79 & 9,21 & \\
\hline Segundo quintil & 92,73 & 7,27 & \\
\hline $\begin{array}{l}\text { Quintil intermedio (Medianamente } \\
\text { pobre) }\end{array}$ & 93,99 & 6,01 & \\
\hline Cuarto quintil & 92,16 & 7,84 & \\
\hline Quintil superior (Muy rico) & 94,02 & 5,98 & \\
\hline Región & & & 0,0014 \\
\hline Amazonas & 92,60 & 7,40 & \\
\hline Áncash & 93,24 & 6,76 & \\
\hline Apurímac & 91,54 & 8,46 & \\
\hline Arequipa & 95,66 & 4,34 & \\
\hline Ayacucho & 93,62 & 6,38 & \\
\hline Cajamarca & 91,07 & 8,93 & \\
\hline Prov, Const, Del Callao & 93,25 & 7,65 & \\
\hline Cusco & 93,10 & 6,90 & \\
\hline Huancavelica & 92,82 & 7,18 & \\
\hline Huánuco & 91,83 & 8,17 & \\
\hline Ica & 94,06 & 5,94 & \\
\hline
\end{tabular}




\begin{tabular}{|c|c|c|c|}
\hline Junín & 92,22 & 7,78 & \\
\hline La Libertad & 91,37 & 8,63 & \\
\hline Lambayeque & 92,50 & 7,50 & \\
\hline Lima & 93,97 & 6,03 & \\
\hline Loreto & 88,54 & 11,46 & \\
\hline Madre de Dios & 93,62 & 6,38 & \\
\hline Moquegua & 94,74 & 5,26 & \\
\hline Pasco & 90,85 & 9,15 & \\
\hline Piura & 92,07 & 7,93 & \\
\hline Puno & 91,47 & 8,53 & \\
\hline San Martín & 93,18 & 6,82 & \\
\hline Tacna & 95,93 & 4,070 & \\
\hline Tumbes & 91,18 & 8,820 & \\
\hline Ucayali & 90,61 & 9,39 & \\
\hline Área de residencia & & & 0,0421 \\
\hline Urbana & 93,03 & 6,97 & \\
\hline Rural & 91,84 & 8,16 & \\
\hline \multicolumn{4}{|c|}{ Riesgos médicos anteriores al embarazo } \\
\hline Orden de nacimiento & & & 0,0015 \\
\hline Primer nacimiento & 92,74 & 7,26 & \\
\hline Segundo nacimiento & 93,94 & 6,06 & \\
\hline Tercer nacimiento & 92,58 & 7,42 & \\
\hline Cuarto nacimiento & 91,72 & 8,28 & \\
\hline Quinto a más nacimientos & 89,56 & 10,44 & \\
\hline Talla materna (media \pm IC 95\%) & $\begin{array}{c}152,25(152,07- \\
152,43)\end{array}$ & $\begin{array}{c}151,26 \\
150,80-151,7)\end{array}$ & $<0,0001$ \\
\hline Menor a $145 \mathrm{~cm}$ & 88,96 & 11,04 & \\
\hline Mayor o igual a $145 \mathrm{~cm}$ & 93,11 & 6,89 & \\
\hline Aborto & & & 0,0859 \\
\hline Presencia & 91,67 & 8,33 & \\
\hline Ausencia & 93,03 & 6,97 & \\
\hline \multicolumn{4}{|c|}{ Riesgos médicos actuales en el último embarazo } \\
\hline Periodo intergenésico & & & 0,0291 \\
\hline Menor a 24 meses & 90,55 & 9,45 & \\
\hline Mayor de 24 meses & 92,89 & 7,11 & \\
\hline \multicolumn{4}{|c|}{ Riesgos conductuales y ambientales } \\
\hline Suplementación con hierro & & & 0,0007 \\
\hline Presencia & 92,98 & 7,02 & \\
\hline Ausencia & 89,67 & 10,33 & \\
\hline$\overline{\text { Altitud }}$ & & & 0,0587 \\
\hline Menos de 1000 & 93,11 & 6,89 & \\
\hline De 1000 a 1999 & 92,69 & 7,31 & \\
\hline De 2000 a 2999 & 92,62 & 7,38 & \\
\hline De 3000 a 3999 & 91,48 & 8,52 & \\
\hline Mayor o igual a 4000 & 89,86 & 10,14 & \\
\hline
\end{tabular}


En la regresión de Poisson que evalúa la asociación entre el número de controles prenatales y peso al nacer podemos observar que aquellas que a medida que aumenta el rango de controles prenatales disminuye el riesgo de BPN tanto en el modelo crudo (6-7 controles: RPc=0,65, IC 95\%: 0,53-0,80; 8-9 controles: RPc=0,26, IC 95\%: 0,21-0,31; 11 a más controles: $\mathrm{RPc}=0,22$, IC 95\%: 0,18-0,28) como ajustado (6-7 controles: $\mathrm{RPa}=0,65$, IC 95\%: 0,53-0,81; 8-9 controles: $\mathrm{RPa}=0,25$, IC 95\%: 0,21-0,32; 11 a más controles: $\mathrm{RPa}=0,23$, IC 95\%: 0,18-0,29).

Tabla 2. RP crudo y ajustado de asociación entre número de controles prenatales y bajo peso al nacer

\begin{tabular}{ccc} 
Indicador & $\begin{array}{c}\text { Cruda } \\
\text { RP }(\text { IC 95\%) }\end{array}$ & $\begin{array}{c}\text { Ajustada } \\
\text { RP (IC 95 \%) }\end{array}$ \\
\hline Controles prenatales & & \\
$0-5$ controles & 1,00 & 1,00 \\
$6-7$ controles & $0,65(0,53-0,80)$ & $0,65(0,53-0,81)$ \\
$8-10$ controles & $0,26(0,21-0,31)$ & $0,25(0,21-0,32)$ \\
11 a más & $0,22(0,18-0,28)$ & $0,23(0,18-0,29)$ \\
\hline
\end{tabular}

* Ajustado por las variables sexo, edad de la madre, estado conyugal, nivel de educación, quintil de riqueza, orden de nacimiento, aborto, periodo intergenésico, talla materna, suplementación con hierro, altitud, área de residencia y departamento 


\section{DISCUSIÓN}

En nuestro estudio encontramos que el número de controles prenatales estuvo asociado al $\mathrm{BPN}$, es decir a mayor número de controles menor riesgo de BPN. Incluso después de ajustar por todas las variables confusoras la asociación se sigue manteniendo con la misma intensidad. El control prenatal es una práctica recomendada por la OMS, como una medida para identificar oportunamente riesgos en la gestación, promover un crecimiento del niño y evitar complicaciones en la madre y el feto (45).

Nuestros resultados coinciden con lo encontrado por otros autores. Así tenemos que, Zeleke BM et al., encontraron que las madres que no asistieron a los controles prenatales estuvieron casi tres veces más propensas de tener bebés con BPN con respecto a aquellas que tuvieron al menos un control prenatal. Así mismo, por cada visita adicional hubo una reducción del $21 \%$ en el riesgo de tener un bebé con BPN. Esto se atribuye a la asesoría nutricional, atención médica y suplementación ofrecida (58). Otro estudio realizado en Brasil también encontró la misma asociación y mencionan que el control influye en el peso al nacer, porque incrementa la edad gestacional y por ende mejora el peso al nacer. Además, ayuda a identificar riesgos oportunamente para disminuir el riesgo de BPN (59).

La carencia de controles prenatales dificulta la detección de embarazos de riesgo elevado, lo que puede afectar por un lado la salud y nutrición de la madre y esto pueda afectar peso al nacer. El adecuado número de controles prenatales puede afectarse por diversos factores como: brechas culturales y las limitaciones económicas del hogar (6). Un estudio indica que la visita prenatal más importante es la primera, por ello es importante que se realice temprano en el embarazo. Puesto que, los datos médicos reunidos durante dicho control ayudan a identificar a las mujeres con mayor riesgo de parto prematuro y de tener un niño con BPN asociado, por lo que estas mujeres pueden por un lado mejorar el seguimiento y capacitar en señales de alarma para actuar oportunamente.. Por otro lado, hacen hincapié que el control prenatal no puede controlar las diferencias socioeconómicas y ambientales que dan muchas veces como resultado un mal pronóstico de parto. Sin embargo, los controles han demostrado ser muy importantes en la identificación de factores que afectan el resultado del nacimiento, como consumir alcohol, fumar cigarrillos, consumo de drogas, dieta deficiente, entre otros (52). 
En este estudio se obtuvo que el nivel de educación más bajo, peor quintil de riqueza, región natural (sierra y selva) y área de residencia (rural) se asociaron con una mayor probabilidad de tener un bebé con BPN. Este resultado ha sido descrito por múltiples autores (60-62). Madres que con menor nivel de educación y menores ingresos suelen tener una dieta deficiente y son más propensas a adherirse a los mensajes de salud dadas por su contexto social y/o por el conocimiento cognitivo que la educación ofrece. Dai LL et al, encontraron que el nivel de educación de la madre y el número de visitas de atención prenatal podría protegerlas del BPN, puesto que las mujeres con mayor nivel de educación cambian más fácilmente sus factores biológicos, psicosociales y de comportamiento que influyen en el embarazo (61). El nacer en la costa y en área urbana es un reflejo de mejores condiciones de vida como saneamiento ambiental, nutrición, educación, acceso a establecimientos de salud y salarios $(\mathbf{1 9}, \mathbf{6 3})$. Sin embargo, no todos los autores encontraron tal relación, Fallah R et al y Márquez-Beltrán MF et al, describen que el bajo peso fue mayor en el área urbana y lo atribuyen a actividades más rigurosas de cuidado de la madre y educación sanitaria, y factores vinculados con el estilo de vida como mayor exposición a estrés y patrones alimentarios inadecuados, respectivamente $(64,65)$.

Con el fin de disminuir la brecha de accedo a la salud, el MINSA posee disposiciones sobre intervenciones con el fin de reducir la morbilidad y mortalidad neonatal, la cual promueve que los más pobres y desprotegidos puedan acceder de manera prioritaria a los servicios de salud y procura que exista una adecuada distribución de recursos humanos y materiales en los centros de salud y en la comunidad. Además, se llevan a cabo procesos prestacionales en los que se especifica que las Direcciones de Salud (DISAS) y Direcciones Regionales de Salud (DIRESAS) o su similar en las regiones deben llevar a cabo intervenciones que se ajusten a la realidad geográfica y sociocultural. Si la zona es de difícil acceso geográfico, como las zonas rurales y dispersas, las intervenciones se deben realizar en el hogar y en la comunidad por medio de visitas familiares integrales (en grupo) (66). Por otro lado, se pueden realizar actividades de captación y seguimiento, en especial de gestantes que en su primer trimestre no hayan asistido a su primer control prenatal. Para esto se realizan visitas domiciliarias con el fin de brindar orientación y sensibilizar a la madre y su familia sobre la importancia de las atenciones prenatales reenfocadas. En las visitas se proporciona consejería y seguimiento del estado nutricional, educación sobre la identificación de signos de alarma, control de los movimientos del 
feto, importancia de la toma de pruebas de laboratorio, verificación del consumo de suplementos maternos y/o medicamentos prescritos, verificación de vacunas, identificación de violencia de género y firma de compromiso para asistir a sus siguientes controles. En este proceso, los agentes comunitarios son de gran utilidad (39).

Perú es un país con un gran número de personas que viven a gran altitud sobre el nivel del mar. Este estudio encontró que la prevalencia de BPN aumenta en personas que viven a mayor número de m.s.n.m. Un estudio realizado en Perú encontró el mismo resultado (36), situación similar a la encontrada en otros países (67). Esta asociación se explica por la disminución de la presión parcial del oxígeno, conocida como hipoxia hipobárica que provoca disminución de la saturación de oxígeno arterial e induce disminución del crecimiento celular. Esta hipoxia fetal se ve reflejada en su mayoría en mujeres que residen en lugares con altitudes mayores a 2500 m.s.n.m (35). Es a partir de esta altura que la saturación de oxígeno disminuye gradualmente y se produce un menor flujo úteroplacentario lo que puede traer consigo un aumento de hemoglobina que genera una mayor viscosidad en sangre y una menor disponibilidad de glucosa, traduciéndose en un retardo de crecimiento intrauterino y por consiguiente, niños más pequeños y con bajo peso (68). Aunque, también cabe resaltar, que en nuestro país y en los países que tienen población que viven a gran altitud, las condiciones de acceso, pobreza y condiciones de vida en general suelen ser más limitadas que los que viven a nivel del mar $(\mathbf{6 9}, \mathbf{7 0})$.

Algunos países que viven en estas condiciones de altitud son Bolivia, Ecuador y Colombia. La atención integral de la gestante en Bolivia procura brindar a toda mujer en la red de servicios de salud: control prenatal y en el puerperio, consulta ginecológica, atención ginecológica, entre otros. El mínimo de controles prenatales es cuatro, donde el primer control debe realizarse antes de las 22 semanas. En los controles prenatales debe realizarse el control de riesgo obstétrico, detección de complicaciones, promoción de la lactancia materna, suplementación (hierro, ácido fólico y vitamina C) y entrega de materiales educativos. Además, a la mujer embarazada se le realizará examen clínico general, examen de mamas, examen obstétrico (determinación de edad gestacional), evaluación del crecimiento fetal y la altura uterina, examen ginecológico (toma de muestra de Papanicolau), medición y registro de la presión arterial, medición de peso y talla materna (cálculo de IMC), determinación de la hemoglobina o signos clínicos de anemia, determinación de grupo sanguíneo y factor $\mathrm{Rh}$, determinación de sífilis, examen de orina, detección de glicemia en sangre y orientación (71). En Ecuador, el control 
prenatal de la gestante tiene la finalidad de evaluar el desarrollo normal del embarazo y salud del neonato mediante la detección de riesgos y enfermedades en el embarazo. Este control debe ser brindado por personal capacitado y en colaboración del equipo de salud y médico gineco-obstetra. Además, la atención prenatal debe estar al acceso de todas las mujeres gestantes, de manera individual, regida según el entorno en el que vive y con continuidad. El primer control se ha de realizar en el primer trimestre de embarazo y en total deben completarse como mínimo cinco controles prenatales, en los cuales se realiza el cálculo de la fecha probable de parto, medición y registro de la presión arterial, medición y registro de peso y talla (cálculo de IMC materno), determinación de factores de riesgo, descarte de incompatibilidad de grupo y factor $\mathrm{Rh}$, descarte de anemia, detección de VIH y hepatitis $\mathrm{B}$, tamizaje de función tiroidea, toma de citología cérvico vaginal, examen odontológico, entrega de información pertinente a la gestante (cambios fisiológicos del embarazo, higiene personal, actividad física durante el embarazo, educación nutricional, educación prenatal y planificación familiar, signos de alarma, signos de alarma para preeclampsia, infección obstétrica, parto pretérmino) (72). El control prenatal en Colombia permite identificar la salud de la madre y el bebé, este control médico integral incluye consultas de salud, exámenes clínicos, ecografías y reconocimiento de posibles problemas en la gestación (73). El número y frecuencia de controles dependerá de cada gestante (nulípara: diez visitas, multípara: siete visitas) y se recomienda que el primer control sea antes de las 10 semanas de gestación (74). En el control prenatal, el encargado de salud anotará aquellos datos básicos de interés (edad, ocupación, lugar de nacimiento y residencia, hábitos de alimentación, hábitos nocivos, violencia intrafamiliar, antecedentes de embarazos y partos, antecedentes de enfermedades generales y cirugías), realizará exámenes para asegurar normalidad del embarazo, crecimiento y desarrollo del bebé, se realizará mediciones de peso, talla y su presión arterial y altura uterina. Las gestantes asisten a consultas individuales y a programas de educación grupales (73). Lo que podemos ver, es que los países de la región que comparten áreas a gran altitud (Bolivia, Colombia y Ecuador), dentro de sus políticas de atención a la gestante adoptan diferentes números de controles prenatales. Sin embargo, en ninguno de los países se hace diferencias por nivel de altitud.

La baja talla materna también estuvo asociada al BPN. Otros estudios refuerzan este resultado y mencionan que una talla baja aumenta el riesgo de BPN, pudiendo duplicar o 
inclusive cuadruplicar éste (75-77). La talla alcanzada por la madre es un reflejo del nivel socioeconómico, educación y condiciones de vida, sobre todo en la primera infancia (78).

En el caso del orden de nacimiento y el periodo intergenésico (PI), a mayor número de hijos y menor intervalo entre nacimientos aumenta el riesgo de BPN. Algunos autores mencionan que el BPN es mayor en el primer y segundo nacimiento, luego disminuye en el tercero y aumenta nuevamente en el cuarto o quinto nacimiento $(\mathbf{2 0}, \mathbf{3 1}, \mathbf{7 9})$. En relación con el PI, no solo el corto sino el periodo largo aumenta el riesgo $(\mathbf{8 0 ,}, \mathbf{8 1})$. La asociación entre corto PI y BPN ha sido explicado por el agotamiento de la nutrición materna, agotamiento de las reservas de hierro y ácido fólico y el estrés postparto (79). Hay un desequilibrio nutricional entre feto - madre dándose una competencia biológica (82). Por otro lado, el BPN en relación al PI prolongado puede darse por la disminución gradual de la adaptación fisiológica y anatómica del sistema reproductivo después de un tiempo prolongado si la mujer no concibe otro feto, así mismo si aumenta el PI hay coexistencia de complicaciones maternas como hipertensión, obesidad, preeclampsia y diabetes, complicaciones que pueden afectar el peso al nacer (83).

Otro resultado obtenido fue a la presencia de abortos y BPN (aunque marginalmente significativa). Situación similar a la descrita por otros autores, que mencionan que a mayor número de abortos mayor el riesgo de BPN (84). No se analizaron el número de abortos y no se tiene información del tipo de aborto (inducido o natural). En relación a esto, no se tiene suficiente evidencia y no se conoce si los riesgos adversos en el embarazo son un confusor o es una respuesta a los procedimientos realizados (específicamente por daños ocasionados por abortos inducidos) $(\mathbf{8 5})$.

La anemia en el embarazo es uno de los factores que se han asociado en los resultados adversos del embarazo como: nacimientos prematuros, BPN, mortalidad materna y perinatal. El riesgo de las madres anémicas en tener un bebé con BPN fue casi dos veces mayor que las madres no anémicas (86). Por otro lado, se ha encontrado que por cada aumento de $1 \mathrm{~g} / \mathrm{dl}$ en el nivel de hemoglobina de la madre, aumenta el peso al nacer en $44 \mathrm{~g}$ (87). Sin embargo, no se conocen las causas de este resultado. Por otro lado, la alta prevalencia de anemia materna se atribuye a la baja ingesta dietética de hierro y ácido fólico, la biodisponibilidad de hierro o la pérdida crónica de sangre debido a infecciones (88). Haider B et al, evaluaron la asociación de anemia materna y uso prenatal de hierro con los resultados adversos del embarazo. En dicho estudio, encontraron que la 
suplementación de hierro (hasta $66 \mathrm{mg} /$ día) se asoció a un aumento en el peso al nacer y una disminución en el riesgo de BPN (89).

Una de las limitaciones de nuestro estudio es que la ENDES-2016 y nuestro estudio son de diseño transversal por lo que no se puede afirmar que las variables "asociadas" son causales. También, la información dada depende en parte de lo que recuerdan las encuestadas, en la que existe la probabilidad de olvido o el sesgo de memoria. Dentro de sesgos de memoria, podríamos mencionar que el peso al nacer puede no ser tomado de igual manera en toda la población, así tenemos que por ejemplo en zonas rurales en donde hay mayor probabilidad de parto domiciliario, el peso registrado en el CRED (carné de crecimiento y desarrollo), podría no corresponder con el peso al nacer, sino con el primer peso que se realizó en el establecimiento (podría ser el peso del niño de un mes de nacido). Aunque cabe mencionar, que en los últimos años los partos domiciliarios se han reducido grandemente (en el año 2009 fue de 18,7\% y al año 2017 fue de 7,0\%) (90). Así mismo, como limitación en la recolección de data es que se tomó como variable de resultado el BPN y no RCIU. El no tener en conocimiento de la edad gestacional de la madre al momento del parto, podría tomar a los RCIU como BPN cuando en realidad no lo son. Por otro lado, no se tuvo información de enfermedades de la madre en su último embarazo (cáncer, VIH-SIDA, diabetes, eclampsia entre otros) que podrían haber afectado el peso al nacer y número de semanas de gestación y por ende haber influido en el número de controles prenatales. Además, otra limitación referida al número de controles prenatales, es que solo refleja la cantidad pero, no se indica la calidad de los mismos, ni espaciamiento de las visitas; y esto puede haber sido diferente de acuerdo al lugar y nivel socioeconómico.

También, el no tener en conocimiento el nivel de atención de salud donde se dieron los controles prenatales puede significar una limitación, puesto que un estudio determinó que existe mayor mortalidad neonatal para recién nacidos con BPN nacidos en hospitales que son atendidos en establecimientos de nivel I, nivel II y nivel III sin neonatólogos, en comparación con los nacidos en centros de nivel III con equipo multidisciplinario (91). Otro estudio, realizó un metaanálisis donde concluyeron que los recién nacidos BPN y nacidos muy prematuros en hospitales de niveles más bajos tenían un riesgo mayor de mortalidad neonatal (92). Además, una limitación de nuestro estudio es que podría haber causalidad reversa, debido a que los niños que nacieron a término tuvieron mayor 
oportunidad de tener más controles prenatales, que aquellos niños que nacieron antes con peso aceptable.

Las fortalezas de nuestro estudio es que está basado en una encuesta a nivel nacional, la cual es representativa a nivel país; y el tamaño de muestra que fue lo suficientemente grande. Además, es una encuesta cuyos encuestadores son entrenados y estandarizados para realizar la toma de datos. 


\section{CONCLUSIÓN}

Se encontró que el aumento de número de controles prenatales disminuye el riesgo de BPN. Esto coincide con la recomendación del nuevo modelo de atención prenatal OMS que establece ocho controles como mínimo. Siguiendo estas recomendaciones, la diferencia de dos controles a más significa mayor oportunidad para detectar y gestionar los posibles problemas en la gestante; además de reforzar la comunicación entre el personal de salud y la gestante. Por otro lado, la recomendación de la OMS que establece que el primer contacto con la gestante debe ser antes de las 12 semanas en comparación a las 14 semanas en Perú. Esto puede ayudar a identificar oportunamente riesgos que podrían afectar tanto a la salud de la madre como del niño. Así mismo, es importante continuar con las disposiciones generales de la atención integral en salud materna como la suplementación, ya que estas forman parte de las estrategias de intervención nutricional en la gestante. Además, se sugiere realizar estudios posteriores que evalúen la semana de gestación en la que se da el primer control prenatal, la edad gestacional al momento del parto, la calidad y contenido de la atención prenatal; aspectos que ayudarán a controlar los resultados y a identificar de manera más precisa el número de controles prenatales que inciden en el peso al nacer. 


\section{REFERENCIAS BIBLIOGRÁFICAS}

1. OMS: Organización Mundial de la Salud. [Internet] Suiza: OMS; 2015 [citado 20 de marzo de 2018]. Estadísticas Sanitarias Mundiales 2005. Disponible en: http://www.who.int/healthinfo/statistics/whostat2005es2.pdf

2. Hughes M., Black R., Katz J. 2500-g Low Birth Weight Cutoff: History and Implications for Future Research and Policy. Maternal and Child Health Journal. 2017;21(2):283-289.

3. Pan American Health Organization, Worl Health Organization. CORE INDICATORS Health Situation in the Americas 2017 [Internet]. Washintong, D.C; 2017 [citado 24 de agosto de 2018] p. 4-19. Disponible en: https://reliefweb.int/sites/reliefweb.int/files/resources/PAHO\%20CoreIndicators20 17 eng.pdf

4. United Nations Children's Fund. MPROVING CHILD NUTRITION The achievable imperative for global progress [Internet]. New , NY; 2013 abr [citado 24 de agosto de 2018] p. 1-129. Disponible en: https://data.unicef.org/wpcontent/uploads/2015/12/NutritionReport_April2013_Final_29.pdf

5. Hack M., Klein N., Taylor H. Long-term developmental outcomes of low birth weight infants. Future Child. 1995; 5(1): 176-196.

6. Rendón M., Apaza D., Vildoso M. Incidencia y factores de riesgo de bajo peso al nacer en poblacion atendida en hospitales del Ministerio de Salud del Perú. Ginecología y Obstetricia de México. 2012;10.

7. Baños G. Factores de riesgo asociados al bajo peso al nacer. Revista Cubana de Salud Pública. junio de 2012;38(2):238-45.

8. Organización Mundial de la Salud. Metas mundiales de nutrición 2025: Documento normativo sobre bajo peso al nacer [Internet]. Organización Mundial de la Salud; 
2017 [citado 2 de abril de 2018]. Disponible en: http://apps.who.int/iris/bitstream/handle/10665/255733/WHO_NMH_NHD_14.5_s pa.pdf?sequence $=1$

9. UNICEF. Progreso para la Infancia - Bajo peso al nacer [Internet]. 2006 [citado 2 de abril de 2018]. Disponible en: https://www.unicef.org/spanish/progressforchildren/2006n4/index_lowbirthweight. $\underline{\mathrm{html}}$

10. Coronel C., Rivera I. Peso bajo al nacer, su influencia en la salud durante el primer año de vida. Rev Mex Pediatr. 2003;70(6): 283-287.

11. Peraza G., Pérez S., Figueroa Z. Factores asociados al bajo peso al nacer. Rev Cubana Med Gen Integr 2001; 17(5): 490-6.

12. Herrera A., Rodríguez J., Suárez R., Hernández V. El sistema inmune neonatal y su relación con la infección. Alergia, Asma e Inmunología Pediátricas. 2013; 22 (3): 101-113.

13. Álvarez R., Urra L., Aliño M. Repercusión de los Factores de Riesgo en el Bajo Peso al Nacer. RESUMED. 2001;14(3):117-24.

14. Paisán L., Sota I., Muga O., Imaz M. El recién nacido de bajo peso. Hospital Donostia. San Sebastián. UPV. Departamento de Medicina. Unidad Docente de San Sebastián. Protocolos Diagnóstico Terapéuticos de la AEP: Neonatología. 2008; 9:78-84.

15. Larroque B., Bertrais S., Czernichow P., Léger J. School Difficulties in 20-Year-Olds Who Were Born Small for Gestational Age at Term in a Regional Cohort Study. Pediatría. 2001 Jul; 108 (1): 111-115.

16. Kranzler J., Rosenbloom A., Martinez V., Guevara-Aguirre J. Normal intelligence with severe insulin-like growth factor I deficiency due to growth hormone receptor deficiency: a controlled study in a genetically homogeneous population. J Clin Endocrinol Metab. 1998; 83:1953-1958.

17. Cacicedo L., Sánchez F. Sistema GH-IGF-I y envejecimiento cerebral. Endocrinol Nutr. 2010; 57(6):235-239. 
18. Valero J., Soriano T., Albaladejo R., et al. Risk factors for low birth weight: A review. European Journal of Obstetrics Gynecology and Reproductive Biology, 2004; 116 (1):3-15 .

19. Committee to Study the Prevention of Low Birthweight; Division of Health Promotion and Disease Prevention; Institute of Medicine. Preventing Low Birthweight. National Academies Press. 1985.

20. Quintana V., Inés N., Zárraga M., Luis J., Ávila Reyes R. Recién nacidos con bajo peso; causas, problemas y perspectivas a futuro. Boletín médico del Hospital Infantil de México. febrero de 2004;61(1):73-86.

21. Mumbare S., Maindarkar G., Darade R, et al. Maternal risk factors associated with term low birth weight neonates: a matched-pair case control study. Indian Pediatr. 2012; 49(1):25-28.

22. Mortensen L. Socioeconomic inequality in birth weight and gestational age in Denmark 1996-2007: using a family-based approach to explore alternative explanations. Soc Sci Med, 2013;76(1):1-7.

23. Khan J., Islam M., Awan N., Muurlink O. Analysis of low birth weight and its covariants in Bangladesh based on a sub-sample from nationally representative survey. BMC Pediatrics, 2018;18:100.

24. Souto S., Santos F., Coca L.. Nascimento de recém-nascidos de baixo peso em instituição filantrópica terciária do Município de Piracicaba. Enferm. Glob,2011;10: 23

25. Ulloa A., Del Castillo A., Moreno Marco. Factores de riesgo asociados a bajo peso al nacimiento. Rev Hosp Jua Mex 2016;83(4): 122-128

26. Espinosa G., Aurelio M., Saenz L., Escobar C., Andrés J. Factores de riesgo del bajo peso al nacer, Hospital Gineco-Obstétrico Provincial de Sancti Spíritus, Cuba. Rev panam salud pública. agosto de 1999;6:95-8. 
27. Sojo L, et al. Are birth weight predictors in diabetic pregnancy the same in boys and girls?. European Journal of Obstetrics and Gynecology and Reproductive Biology, 2010;153(1), $32-37$

28. Álvarez D, Valdés L., et al. El exceso y el bajo peso corporal al nacimiento en hijos de madres con diabetes. Revista Cubana de Obstetricia y Ginecología, 2012;38(3), 294-304.

29. Bakketeig L., Hoffman H., Harley E. The tendency to repeat gestational age and birth weight in successive births. Am J Obstet Gynecol, 1979;135(8):1086-1103.

30. Brooks S, Vicente G, Javier P., Lissette O., et al. Importancia de los antecedentes maternos en el recién nacido bajo peso. Revista Cubana de Medicina General Integral. octubre de 2000;16(5):502-7.

31. Roberto Á., Luis C., Miriam A. Repercusión de los Factores de Riesgo en el Bajo Peso al Nacer. RESUMED. 2001;14(3):117-24.

32. Food and Agriculture Organization of the United Nations. Necesidades de vitamina A, hierro, folato y vitamina B12: informe de una Consulta Mixta FAO/OMS de Expertos. Food \& Agriculture Org.; 1991. 148 p.

33. Hamułka J., Zielińska M., Chądzyńska K. The combined effects of alcohol and tobacco use during pregnancy on birth outcomes. Rocz Panstw Zakl Hig 2018;69(1):45-54.

34. Zahran S., Breunig I., Link B., Snodgrass J., Weiler S. Quasi-Experimental Analysis of Maternal Altitude Exposure and Infant Birth Weight. American Journal of Public Health. 2014;104(1):166-174.

35. Grandi C., Dipierri J., Luchtenberg G., Morezco A., Alfaro E. Efecto de la altitud sobre el peso al nacer y eventos perinatales adversos en dos poblaciones argentinas. Revista Facultad de Ciencias Medicas. 2013;70(2):55-62.

36. Villamonte W., Jerí M., Lajo L., Monteagudo Y., Diez G. Birth weight at term at different altitudes in Peru. Rev. peru. ginecol. obstet. 2011: 57(3):144-150. 
37. Instituto Nacional de Estadística e Informática. INEI - Perú: Encuesta Demográfica y de Salud Familiar 2016 - Nacional y Regional [Internet]. [citado 2 de abril de 2018]. 539

p.

Disponible

en:

https://www.inei.gob.pe/media/MenuRecursivo/publicaciones_digitales/Est/Lib1433 lindex.html

38. Ministerio de Salud. Directiva Sanitaria de Suplementación con micronutrientes para los niños (as) menores de 5 años, gestantes y puerperas. [Internet] Ministerio de Salud; 2013 [citado 08 de octubre de 2018]. Disponible en: http://www.diresacusco.gob.pe/salud_individual/normas/DIRECTIVA\%20SANITA RIA\%20MICRONUTRIENTES\%20Curvas.pdf

39. Ministerio de Salud. Norma Técnica de Salud para la atención integral de salud materna (NTS ${ }^{\circ} 105$ - MINSA/DGSP.V.01). [Internet] Ministerio de Salud; 2013 [citado 27 de abril de 2018]. Disponible en: http://www.unfpa.org.pe/Legislacion/PDF/20131224-MINSA-NT-Atencion-SaludMaterna.pdf

40. Ministerio de Salud. Norma Técnica - Manejo terapéutico y preventivo de la Anemia en niños, adolescentes, mujeres gestantes y puérperas. [Internet] Ministerio de Salud; 2013 [citado 08 de octubre de 2018]. Disponible en: http://bvs.minsa.gob.pe/local/MINSA/4190.pdf

41. Ministerio de Salud. Resolución Ministerial 159 - 2014 /MINSA [Internet]. 2014 [citado 4 de octubre de 2018]. Disponible en: http://www2.saludmoquegua.gob.pe/web/images/Programas/Materno/kRM1592014 modificaciones\%20normas\%20tecnicas\%20atencion\%20integral\%20materna.pdf

42. Ministerio de Salud. Norma Técnica de Salud para la Implementación del Listado Priorizado de Intervenciones Sanitarias Garantizadas para la Reducción de la Desnutrición Crónica Infantil y Salud Materno Neonatal [Internet]. Servicios Industriales \& Editores SAC; 2008 [citado 27 de septiembre de 2018]. Disponible en: http://bvs.minsa.gob.pe/local/MINSA/1078_DGSP259.pdf

43. Organización Mundial de la Salud. Recomendaciones de la OMS sobre atención prenatal para una experiencia positiva del embarazo [Internet]. Ediciones de la OMS; 
2016 [citado 24 de agosto de 2018]. Disponible en: http://apps.who.int/iris/bitstream/handle/10665/250802/WHO-RHR-16.12spa.pdf?sequence $=1$

44. Organización Mundial de la Salud. La OMS señala que las embarazadas deben poder tener acceso a una atención adecuada en el momento adecuado [Internet]. World Health Organization. [citado 24 de agosto de 2018]. Disponible en: http://www.who.int/es/news-room/detail/07-11-2016-pregnant-women-must-beable-to-access-the-right-care-at-the-right-time-says-who

45. Organización Mundial de la Salud. WHO recommendations on antenatal care for a positive pregnancy experience. [Internet] Organización Mundial de la Salud;2016 [citado 22 de agosto de 2018]. Disponible en: http://apps.who.int/iris/bitstream/handle/10665/250796/9789241549912eng.pdf?sequence $=1$

46. American Academy of Pediatrics Committee on Fetus and Newborn and Amercian College of Obstetricians and Gynecologists Committee on Obstetric Practice. Guidelines for Perinatal Care 8th. Kilpatrick SJ : Papile L. 2017.

47. American College of Obstetricians and Gynecologists., Beckmann C., Ling F., Barzansky B., Herbert W., Laube D., et al. Obstetrics and gynecology 6th ed. Philadelphia: Wolters Kluwer Health/Lippincott Williams \& Wilkins. 2010

48. Committee to Study Outreach for Prenatal Care., Division of Health Promotion and Disease Prevention., Institute of Medicine Staff., National Academy of Sciences., Brown S. Prenatal Care: Reaching Mothers, Reaching Infants. Washington, D.C.: National Academies Press. 1988.

49. Carter E., Tuuli M., Caughey A., Odibo A., Macones G., Cahill A. Number of prenatal visits and pregnancy outcomes in low-risk women. Journal of perinatology: official journal of the California Perinatal Association. 2016; 36(3):178-181.

50. American College of Obstetricians and Gynecologists., Society for Maternal-Fetal Medicine. [Internet] ACOG/SMFM;2015 [citado 08 de octubre de 2018]. Disponible en: $\quad$ https://www.acog.org/Clinical-Guidance-and-Publications/Obstetric-CareConsensus-Series/Levels-of-Maternal-Care 
51. Menard M., Kilpatrick S., Saade G., Hollier L., Joseph G., Barfield W., et al. Levels of maternal care. American Journal of Obstetrics and Gynecology. 2015; 212(3):25971.

52. Henderson J. The Cost Effectiveness of Prenatal Care. Health Care Financing Review. 1994;15(4):21-32.

53. Tayebi T., Hamzehgardeshi Z., Shirvani M., Dayhimi M., Danesh M. Relationship between Revised Graduated Index (R-GINDEX) of Prenatal Care Utilization \& Preterm Labor and Low Birth Weight. Global Journal of Health Science. 2014;6(3):131-137.

54. Instituto Nacional de Estadística e Informática. Ficha técnica: Encuesta Demográfica y de Salud Familiar 2016 [Internet]. [citado 30 de abril de 2018]. Disponible en: http://iinei.inei.gob.pe/iinei/srienaho/Descarga/DocumentosMetodologicos/2016$\underline{\text { 5/FichaTecnica.pdf }}$

55. Instituto Nacional de Estadística e Informática. Ficha técnica: Encuesta Demográfica y de Salud Familiar 2016 [Internet]. [citado 30 de abril de 2018]. Disponible en: http://iinei.inei.gob.pe/iinei/srienaho/Descarga/DocumentosMetodologicos/20165/FichaTecnica.pdf

56. Pinzón-Rondón Á., Gutiérrez V., Madriñan H., Amin J, Aguilera-Otalvaro P, HoyosMartínez A. Low birth weight and prenatal care in Colombia: a cross-sectional study. BMC Pregnancy and Childbirth. 2015;15:118.

57. Instituto Nacional de Estadística e Informática. Sistema de Documentación Virtual y Documentaciones Estadisticas [Internet]. [citado 30 de abril de 2018]. Disponible en: https://webinei.inei.gob.pe/anda_inei/index.php/catalog/563/sampling\#page=data_c ollection\&tab=study-desc

58. Zeleke B., Zelalem M., Mohammed N. Incidence and correlates of low birth weight at a referral hospital in Northwest Ethiopia. The Pan African Medical Journal. 2012;12:4. 
59. Wehby G., Murray J., Castilla E., Lopez J., Ohsfeldt R. Prenatal care effectiveness and utilization in Brazil. Health Policy and Planning. 2009;24(3):175-188. doi:10.1093/heapol/czp005.

60. Muula A., Siziya S., Rudatsikira E.. Parity and maternal education are associated with low birth weight in Malawi. African Health Sciences. 2011;11(1):65-71.

61. Dai L., Mao Y., Luo X., Shen Y. Prenatal Care in Combination with Maternal Educational Level Has a Synergetic Effect on the Risk of Neonatal Low Birth Weight: New Findings in a Retrospective Cohort Study in Kunshan City, China. Baradaran HR, ed. PLoS ONE. 2014;9(11):e113377.

62. Li C., Sung F. Socio-economic inequalities in low-birth weight, full-term babies from singleton pregnancies in Taiwan. Public Health. 2008 ;122(3) :243-250

63. INEI: Instituto Nacional de Estadística e Informática. [Internet] Perú: INEI; 2002 [citado 20 de marzo de 2018]. Tendencias de Riesgo de Nacer con Muy Bajo Peso en el Perú y $\quad$ Factores Condicionantes. Disponible en: https://www.inei.gob.pe/media/MenuRecursivo/publicaciones_digitales/Est/Lib0 566/Libro.pdf

64. Fallah R., Kazemnejad A., Zayeri., Shoghli A. Birthweight Related Factors in Northwestern Iran: Using Quantile Regression Method. Global Journal of Health Science. 2016;8(7):116-125.

65. Márquez M., Vargas J., Quiroga E., Pinzón G. An analysis of low birth weight in Colombia, 2005-2009. Rev Salud Publica (Bogota). 2013;15(4):577-588

66. Ministerio de Salud. Norma Técnica de Salud que Establece el Conjunto de intervenciones Articulada para la Reducción de la Mortalidad Neonatal en el Primer Nivel de Atención de Salud, en la Familia y la Comunidad [Internet]. 2009 [citado 10 de octubre de 2018]. Disponible en: http://bvs.minsa.gob.pe/local/minsa/2783.PDF

67. Jensen G., Moore L. The effect of high altitude and other risk factors on birthweight: independent or interactive effects? American Journal of Public Health. 1997;87(6):1003-1007. 
68. Gonzales G. Impacto de la altura en el embarazo y en el producto de la gestación. Revista Peruana de Medicina Experimental y Salud Publica. junio de 2012;29(2):2429.

69. Leonard W. Nutritional determinants of high altitude growht in Nuñoa, Peru. Am J Phys Anthropol. 1989;80(3):341-52.

70. Stinson S. The effect of high altitude on the growth of children of high socioeconomic status in Bolivia. Am J Phys Anthropol. 1982;59(1):61-71.

71. Ministerio de Salud y Deportes. Norma boliviana de salud nb-msd-02-2000 atención a la mujer y el recién nacido en centros de salud, puestos de salud y hospitales básicos de apoyo [Internet]. 2003 [citado 7 de octubre de 2018]. Disponible en: https://srhr.org/abortion-policies/documents/countries/05-Bolivia-GuidelinesWomen-and-Newborn-Health-Care-2003.pdf

72. Ministerio de Salud Pública del Ecuador. Control Prenatal. Guía de Práctica Clínica [Internet]. Primera Edicion. Quito: Dirección Nacional de Normatización; 2015 [citado 9 de octubre de 2018]. Disponible en: https://www.salud.gob.ec/wpcontent/uploads/2014/05/GPC-CPN-final-mayo-2016-DNN.pdf

73. Ministerio de Salud y Protección Social. Guía de Práctica Clínica para la prevención, detección temprana y tratamiento del embarazo, parto o puerperio. [Internet]. Bogotá. Colombia; 2013 [citado 10 de octubre de 2018]. Disponible en: https://www.minsalud.gov.co/sites/rid/Lists/BibliotecaDigital/RIDE/INEC/IETS/GP $\underline{\text { C_Ptes_Embarazo.pdf }}$

74. Ministerio de Salud y Protección Social. Guías de Práctica Clínica para la prevención, detección temprana y tratamiento del embarazo, parto o puerperio.Para uso de profesionales de salud, 2013 [Internet]. Bogotá. Colombia; 2013 [citado 10 de octubre de 2018].

Disponible

en: https://www.minsalud.gov.co/sites/rid/Lists/BibliotecaDigital/RIDE/INEC/IETS/G. Corta.Embarazo.y.parto.Prof.Salud.2013\%20(1).pdf

75. Elshibly E., Schmalisch G. The effect of maternal anthropometric characteristics and social factors on gestational age and birth weight in Sudanese newborn infants. BMC Public Health. 2008;8:244. 
76. Ojha N., Malla D. Low Birth Weight at Term: Relationship with Maternal Anthropometry. JNMA J Nepal Med Assoc. 2007;46(166):52-56

77. Kheirouri S. Alizadeh M. Impact of prenatal maternal factors and birth order on the anthropometric status of newborns in iran. J Biosoc Sci. 2017;49(2):251-264

78. Pajuelo J. El retardo de crecimiento en el Perú [Internet]. Primera. Perú; Enero [citado 24 de agosto de 2018]. 80-98 p. Disponible en: https://www.nestle.com.pe/nutricion/nutrigroup/documents/publicaciones\%20cientif icas\%20-\%20libro\%20dr\%20pajuelo.pdf

79. Barros F., Vitora C., Vaugban J., Estanislau H. BAJO PESO AL NACER EN EL MUNICIPIO DE PELOTAS, BRASIL: FACTORES DE RIESGO. Bol of Saint Panam. 1987;102(6):541-53.

80. Mahande M., Obure J. Effect of interpregnancy interval on adverse pregnancy outcomes in northern Tanzania: a registry-based retrospective cohort study. BMC Pregnancy and Childbirth. 2016;16:140.

81. Adam I., Ismail M., Nasr A., Prins M., Smits L. Low birth weight, preterm birth and short interpregnancy interval in Sudan. J Matern Fetal Neonatal Med. 2009;22(11):1068-71

82. Cecatti J., Correa-Silva E., Milanez H, Morais S., Souza J. The Associations between Inter-Pregnancy Interval and Maternal and Neonatal Outcomes in Brazil. Matern Child Health J. 2008;12(2):275-81.

83. Conde-Agudelo A., Rosas-Bermúdez A., Kafury-Goeta A. Effects of birth spacing on maternal health: a systematic review. Am J Obstet Gynecol. 2007;196(4):297-308.

84. Mandelson M., Maden C., Daling J. Low birth weight in relation to multiple induced abortions. American Journal of Public Health. 1992;82(3):391-394.

85. Hogue C., Cates W., Tietze C. The effects of induced abortion on subsequent reproduction. Epidemiol Rev. 1982;4:66-94.

86. Lone F., Qureshi R., Emanuel F. Maternal anaemia and its impact on perinatal outcome. Trop Med Int Health. 2004;9(4):486-90. 
87. Kaur M., Chauhan A., Manzar M., Rajput M. Maternal Anaemia and Neonatal Outcome: A Prospective Study on Urban Pregnant Women. Journal of Clinical and Diagnostic Research : JCDR. 2015;9(12):QC04-QC08.

88. Suryanarayana R., Chandrappa M., Santhuram A., Prathima S, Sheela S. Prospective study on prevalence of anemia of pregnant women and its outcome: A community based study. Journal of Family Medicine and Primary Care. 2017;6(4):739-743.

89. Haider B., Olofin I., Wang M., Spiegelman D., Ezzati M., Fawzi W. Anemia, prenatal iron use, and risk of adverse pregnancy outcomes: systematic review and metaanalysis. The BMJ. 2013;346:f3443.

90. Instituto Nacional de Estadística e Informática. INEI - Perú: Encuesta Demográfica y de Salud Familiar 2017 - Nacional y Regional [Internet]. 2017 [citado 11 de octubre de 2018]. Disponible en: https://www.inei.gob.pe/media/MenuRecursivo/publicaciones_digitales/Est/Lib1525 /index.html

91. Menard M., Liu Q., Holgren E., Sappenfield W. Neonatal mortality for very low birth weight deliveries in South Carolina by level of hospital perinatal service. Am J Obstet Gynecol 1998;179:374-81.

92. Lasswell S., Barfield W., Rochat R., Blackmon L. Regionalización perinatal para lactantes de muy bajo peso al nacer y muy prematuros: un metanálisis. JAMA 2010; 304: $992-1000$ 


\section{LISTA DE ABREVIATURAS}

ACOG: American College of Obstetricians and Gynecologists

BPN: Bajo Peso al Nacer

CRED: carné de crecimiento y desarrollo

DIRESAS: Direcciones Regionales de Salud

DISAS: Direcciones de Salud

dl: decilitros

EDAs: enfermedades diarreicas agudas

ENDES: Encuesta Demográfica y de Salud Familiar

g: gramos

IGF-I: factores de crecimiento similares a la insulina

IMC: Índice de Masa Corporal

INEI: Instituto Nacional de Estadística e Informática

IRAs: infecciones respiratorias agudas

m.s.n.m: metros sobre el nivel del mar

MEF: mujeres en edad fértil

mg: miligramos

MINSA: Ministerio de Salud

NTS: Norma Técnica de Salud

OMS: Organización Mundial de Salud

PEG: pequeños para la edad gestacional

PI: periodo intergenésico

RCIU: Retardo en el Crecimiento Intrauterino

RPa: razón de prevalencias ajustada

RPc: razón de prevalencias cruda

UPC: Universidad Peruana de Ciencias Aplicadas

vif<3: factor de inflación de la varianza 


\section{ANEXOS}

\section{A. APROBACIÓN DE COMITÉ DE ÉTICA}

icitrito-13

IPE

Charilles, 90 de derilda 2018

Alumnoe

Flor de María Gonzáles Husmán

Wilmar Sogredra Grández

Alumnoc de barrera de Nutriä́n y Dietétio

Universided Penuse de Ciencias Apliadas

Erterent:-

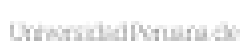

Gonemiplatio

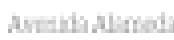

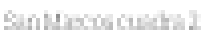

Eenilis

Linith-ping

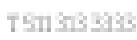

tonsupotips

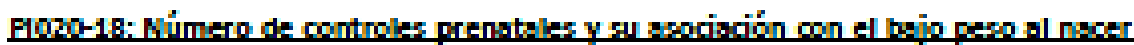
en mujeres de 15 a 49 años según bencuesta demogrática y de selud familiar ENDE2016

Etimsdo[a] invetigodor(s]:

Hemos rocido el protocolo de invetigeción, y loe documentos de soporte, los cusles han sido revisodos en detalle. Luego de ets revisión, se conduy" que ets

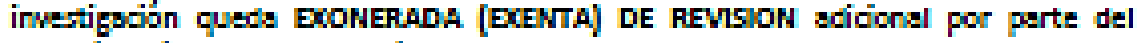

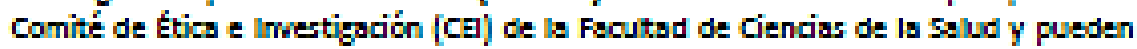
procoder con su ejecuobion. La determiración de ets categorizsoín se bass en lo etablecido en el reglo mento del Comite.

Los invetigodores deben de informer al comité sobre ausuier cambio en el protocolo potteriar a ete ditamen. Del misma modo, de foma anusi y desde ets lechs, los invetigadores deben emiar un breve informe de avenos al comité y un breve informe firal al mamento del cierre definitivo del etudio. Del mismo modo. ante la sperioín de cualquier evento o ersto -previsible a no- que comprometa is integridad y bieneter de be unidsdes de etudio, los inmetigadores o a su equipo de investigoión durante el purso de la implementaión, etos deben de ser también informedos inmediatemente a ete comité a comité ze reserve el derecho de aupervizar de manere inopirada la progreión de is inretigación en avalquier momento y bajo asolquier modalidad. Hos permitimos recordar s los investigodores que la ejsudión de un proyecto de invertigedion que contemple apstos no meritorios de la cotegorizsión de "ekents de revicion" a uns grene falta ls aul pusde er sandionado an el derre defiritivo del estudio e imposibilidad de utilizer cualquier cato recoletedo o generedo en el mismo.

Sin otro partialar, quedo de usted.

Atentamente

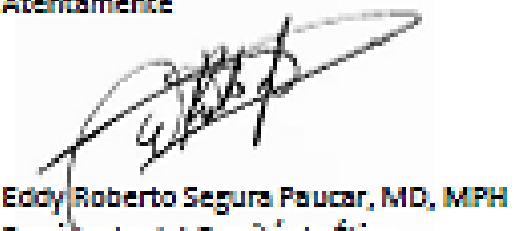

Fresidentecel Comité de Etics

Foculted de ciencias de balud 